\title{
Four Years of Measurements of the Energy Spectrum of Cosmic-Ray-induced-neutrons on the Concordia Antarctic Station Taking into Account Environmental and Systematic Effects
}

HUBERT Guillaume

ONERA DPHY, University of Toulouse

2 avenue Edouard Belin, 31055 Toulouse, France

E-mail: guillaume.hubert@onera.fr

RICAUD Philippe

CNRM, Météo-France / CNRS

42, avenue Coriolis, 31057 Toulouse, France

E-mail: philippe.ricaud@meteo.fr

\begin{abstract}
Neutron spectrometers are operated simultaneously in the framework of a remoted network. The CHINSTRAP, supported by IPEV, aims at recording neutron spectra at the Concordia station $(+3233 \mathrm{~m})$ in Antarctica since December 2105. The neutron spectrometer measures the neutron spectrum over a wide energy range from meV up to tens of $\mathrm{GeV}$ with a short time resolution. Several parameters can influence the measurement: the atmospheric pressure, the hydrometric environment close to the instrument (snowfall) and the atmospheric water vapor. The atmospheric water vapor dominates the ground cosmic ray variations within the first few hundred meters above the ground. The HAMSTRAD polar project is dedicated to measure of the trends in water vapor and temperature profiles from the lower part of the troposphere to the lower part of the stratosphere, using a radiometer. This paper proposes to present data analyses, including short and long-term analyses (first four years) and taking into account environmental and systematic effects. In particular, study focusing on Forbush Decreases and Ground Level Enhancement occurred in September 2017 is proposed.
\end{abstract}

36th International Cosmic Ray Conference -ICRC2019-

July 24th - August 1st, 2019

Madison, WI, U.S.A.

$1 \quad$ Speaker

(C) Copyright owned by the author(s) under the terms of the Creative Commons Attribution-NonCommercial-NoDerivatives 4.0 International License (CC BY-NC-ND 4.0). 


\section{Introduction}

The CHINSTRAP (Continuous High-altitude Investigation of the Neutron Spectra for Terrestrial Radiation Antarctic Project), supported by the French Polar Agency (IPEV), aims at recording neutron spectra at the Concordia station $(+3233 \mathrm{~m})$ since December 2105. Several parameters can influence the measurement: the atmospheric pressure, the hydrometric environment close to the instrument (snowfall) and the atmospheric water vapor. The atmospheric water vapor dominates the ground cosmic ray variations within the first few hundred meters above the ground. Moreover, water vapor contents were recorded in Concordia station in the framework of the HAMSTRAD $\left(\mathrm{H}_{2} \mathrm{O}\right.$ Antarctica Microwave Stratospheric and Tropospheric Radiometers) project. Thus, two contributions were considered to correct count rate data, the air pressure and the atmospheric water vapor.

The four years of measurements performed in Concordia have just been completed. This paper proposes to present data measurements (count rates, energy spectrum, atmospheric water vapor impacts), including short and long-term analyses and taking into account environmental and systematic effects. In particular, study focusing on solar event occurred in September 2017 is proposed.

\section{Description of the CHINSTRAP and HAMSTRAD projects}

The aim of the HAMSTRAD project [1][2] is to measure the trends in water vapour and temperature profiles from the lower part of the troposphere to the lower part of the stratosphere and their links with climate change. The HAMSTRAD radiometer is a genuine state-of-the-art microwave instrument dedicated for the detection of 1) the $60-\mathrm{GHz}$ oxygen line to measure tropospheric temperature profile, and 2) the 183-GHz water vapor $\left(\mathrm{H}_{2} \mathrm{O}\right)$ line to get tropospheric $\mathrm{H}_{2} \mathrm{O}$. The radiometer has been deployed in the Concordia polar station in January 2010 and is working since then. Liquid Nitrogen calibration is performed one or twice per year (summer/winter periods). The instrument is fully automated and data are sent to France on a daily basis. The instrument measures absolute humidity and temperature from the surface to about $10 \mathrm{~km}$ altitude, together with integrated water vapour (IWV), with a time resolution of about 7 minutes. The Liquid Water Path (LWP) parameter is retrieved since 2013, and is sensitive to the presence of supercooled liquid water clouds above the station.

The aim of the CHINSTRAP project is to operate a neutron spectrometer at the Concordia station [3]. The geomagnetic conditions $(\sim 0.001 \mathrm{GV})$ and the high altitude (3233 $\mathrm{m}$ above the sea level) of Concordia let us collect cosmic rays (CRs) data and observe solar particle events (SEP) such as Forbush Decreases (FD) [4] or Ground Level Enhancement (GLE). The neutron spectrometer is based on a Bonner multi-sphere system extended to high neutron energies (energy range from meV to $\mathrm{GeV}$ ). As detailed in previous works, this system was composed of spherical $3 \mathrm{He}$ proportional counters surrounded with spherical PEHD (high density polyethylene) moderators with different thicknesses. Additionally, the spectrometer includes two PEHD spheres with inner tungsten and lead shells in order to increase the response to neutrons above $20 \mathrm{MeV}$. The output pulse signals are treated by a multi-channel analyzer, 
translated as spectrum or count. The response functions were used to convert the measured counting rates to neutron energy spectrum. Fig. 1 presents both instruments, which are operated in the physics shelter of the Concordia station.

(a) Concordia station

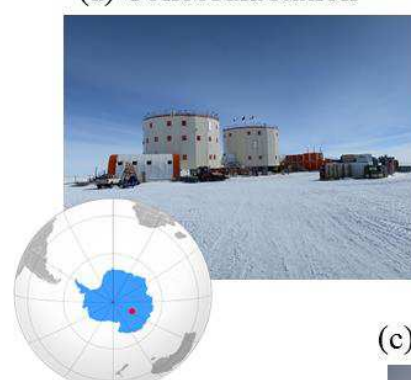

Dome C, Antarctica, Altitude: $3323 \mathrm{~m}$, Cuoff rigidity $\sim 0$ (b) Neutron spectrometer

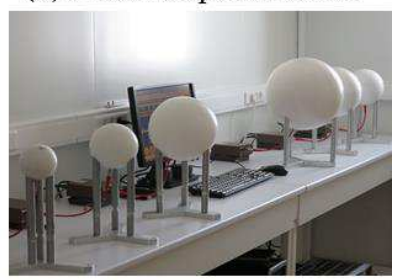

(c) HAMSTRAD radiameter

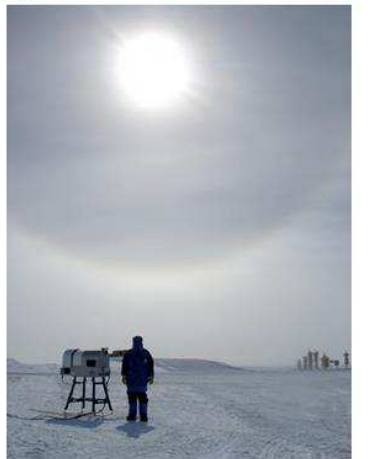

Fig. 1. (a) Concordia station, (b) view of the neutron spectrometer in the Physic shelter and (c) view of the HAMSTRAD radiometer outside the station, it is possible to distinguish a phenomenon of diamond dust producing a halo around the sun from ice crystals in suspension.

\section{Analyses of energy spectrum of CR induced neutrons in Dome C}

Neutron detectors and spectrometers are usually used to monitor the dynamics of incoming cosmic-ray-induced neutrons of high energy (cascade neutron i.e. E > $20 \mathrm{MeV}$ ) under the assumption that local environmental conditions do not influence the neutron fluence rate. Count rates dynamics are mainly due to environmental and systematic effects including the atmospheric pressure variations. Indeed, the cosmic ray flux increases at higher altitude as air pressure and the shielding effect of the atmosphere decrease. More precisely, the vertical distribution of temperature, pressure, density, and composition of the atmosphere constitutes atmospheric structure. These quantities also vary with season and location in latitude and longitude, as well as from night to day; however, under the topic of atmospheric structure, the focus is on the average variations with height above sea level.

Other effect concerns the albedo neutron [5] produced by the interaction of air-shower neutrons with the surface of the Earth. Hydrogen in soil, air and snow determines the amount of ground albedo neutrons in the sensitive energy range from $1 \mathrm{eV}$ to $10 \mathrm{MeV}$. The local environment of the experimental shelter in Concordia is mainly composed by ice water. Precipitation levels are very low in time, providing stable conditions. Albedo contribution does not vary significantly in time and it is not an obstacle to exploit the full energy range of spectra. 


\subsection{Environmental and systematic effects.}

Fig. 2 presents the temporal evolution of the LWP and IWV measured by HAMSTRAD above the altitude of Concordia station from January 2016 to January 2019. In addition, the atmospheric pressure measured in Concordia during the same period using a pressure detector integrated in the neuron spectrometer. Among all contributions, the atmospheric pressure water vapor dominates the ground $\mathrm{CR}$ variations in Concordia [6].

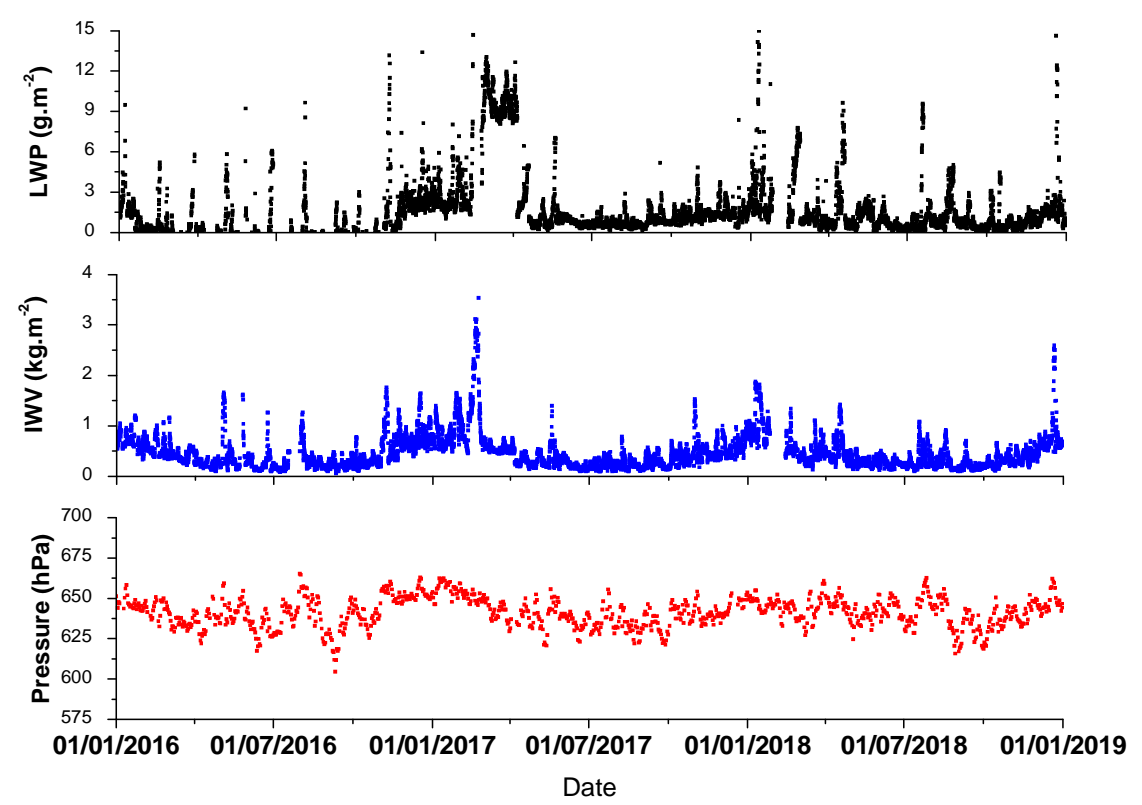

Fig. 2. Temporal evolution of the LWP $\left(\mathrm{kg} \cdot \mathrm{m}^{-2}\right)$ and IWV $\left(\mathrm{g} \cdot \mathrm{m}^{-2}\right)$ as measured by HAMSTRAD above the altitude of Concordia station from January 2016 to January 2019. Also shown are the atmospheric pressure measured in Concordia during the same period thanks to the pressure detector integrated in the neuron spectrometer.

Thus, two contributions were considered to correct count rate data: the atmospheric air pressure and the water vapor. Thus, corrected count rate by air pressure $\mathrm{C}_{\mathrm{P}, \mathrm{corr}}$ is given by the equation 1:

$$
C_{P, \text { corr }}=C_{\text {corr }} \cdot e^{-\beta\left(P_{0}-P\right)}
$$

where $\beta$ is the barometric coefficient $\left(\% \mathrm{hPa}^{-1}\right)$ empirically determined and $\mathrm{C}_{\text {corr }}$ the corrected count rates. The $\beta$ factor, equals to $7.1 \times 10^{-3} \mathrm{hPa}^{-1}$, was empirically determined in a previous work [7]. As described in [6], the atmospheric humidity in the count rate data is taken into account using a single scaling factor $\mathrm{C}_{\mathrm{WV}}$, corr determined by simulations [6]:

$$
C_{W V, \text { corr }}=C \times(1+0.0143 \times \Delta I W V)
$$

where $\triangle \mathrm{IWV}$ corresponds to the difference of the integrated vapor density value with the reference value on the day of the calibration. Concordia environment is well known and stable over time (low precipitation levels). It is one of the driest and coldest places over the world with precipitable water or Integrated Water Vapor (IWV) less than $1 \mathrm{~kg} \mathrm{~m}^{-2}$ over the year reaching less than $0.1 \mathrm{~kg} \mathrm{~m}^{-2}$ in winter [6] with exceptionally wet and warm episodes (IWV >> $1 \mathrm{~kg} \mathrm{~m}^{-2}$ ) as e.g. in January 2017 and December 2019. The atmosphere is considered dry in the Dome C 
when the absolute humidity reaches $0.3 \mathrm{~g} \cdot \mathrm{m}^{-3}$, which is very low compared to values observed in typical moist conditions (i.e. 20 g.m ${ }^{-3}$ at middle latitudes).

Data confirm that the Concordia environment can be therefore considered extremely dry. As shown in a previous work [6], for low atmosphere water vapor content and IWV (in the order of few tenths of $\mathrm{g} . \mathrm{m}^{-3}$ or $\mathrm{kg} . \mathrm{m}^{-2}$ for absolute humidity and IWV, respectively), environmental or systematic effects on fast neutrons are negligible (i.e. a few tenths of a percentage) while they are significant for low-energy neutrons (epithermal and thermal).

\subsection{Modeling approach based on previous works}

Some methods were proposed to model the secondary atmospheric CRs, some of which are based on nuclear transport simulations. The ATMORAD approach [8] combines the GEANT4 simulations of extensive Air Showers to primary GCR spectra define by the ForceField Approximation [9] which provides a simple parametric formula of the differential spectrum. Thus, the whole energy spectrum for protons and $\alpha$-particles can be described using the solar potential $\phi$. This parameter is a formal spectral index whose physical interpretation is not straightforward, especially on short time scales and during periods of active Sun. Several strategies have been developed for the reconstruction of time series of the modulation potential $\phi(t)$, mainly based on neutron monitor data and the yield functions [10].

The secondary CR spectra can be determined as function of the altitude, the longitude, the latitude and the sun activity through the solar potential. In this work, ATMORAD was used to reconstruct the CRs temporal series in Concordia using the solar modulation issued from [10]. The corrected measurements by water vapor and atmospheric pressure effects is compared with the calculated neutron flux using a reference solar potential.

\subsection{Long-term analyses}

Fig. 3 presents the total neutron flux corrected by water vapor and atmospheric pressure effects from January 2016 to January 2019. Experimental data are issued from CHINSTRAP and additional data by calculations using ATMORAD calculations and considering solar potential extract from Oulu data [10]. It is very interesting to note the relevance between corrected measurement and calculations, implying that coupled data issued from HAMSTRAD and CHINSTRAP allow for investigating the dynamic of neutron spectrum in the energy range from $\mathrm{meV}$ to $\mathrm{GeV}$.

The variations are attributable to the solar activity. These trends are also observed on most NMs. The impact of the solar cycle $\left(24^{\text {th }}\right)$ is particularly identifiable on this result. Indeed, this increase reflects the transition from strong to low solar activity. Cycle 24 reached its maximum in April 2014. Reversed polarity polar active sunspot regions observed from December 2016 indicate that the transitional phase to solar cycle 25 is in process. The next solar minimum will be reached probably in late 2019 or 2020. 


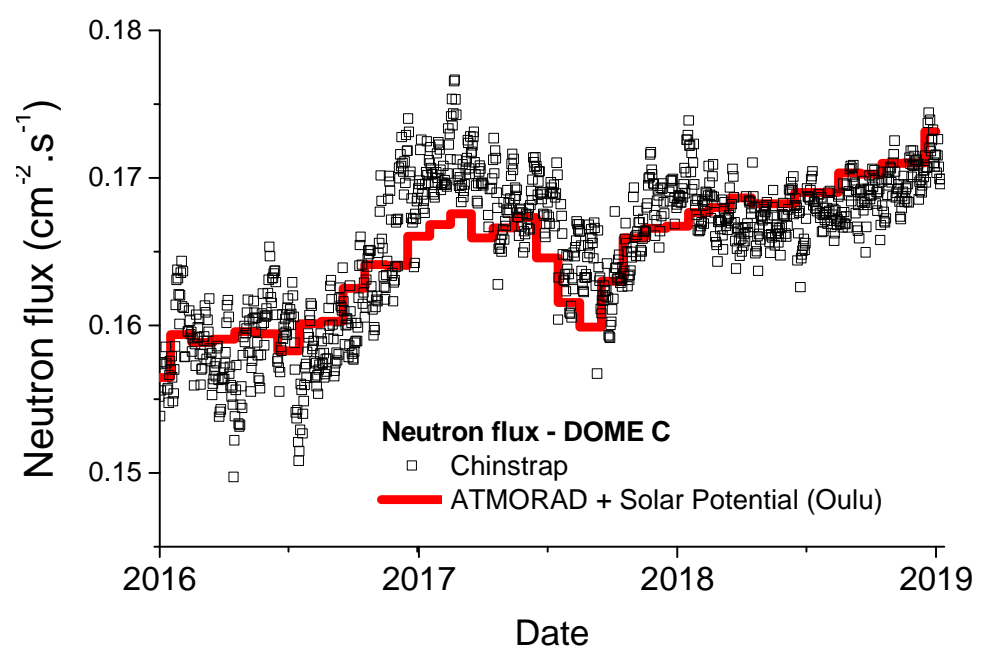

Fig. 3. Total corrected (by water vapor and atmospheric pressure) neutron flux from January 206 to January 2019, issued from measurements and obtained by calculations using ATMORAD calculations and considering solar potential extract from Oulu data.

The daily neutron lethargic spectra (i.e. $\operatorname{Ex} \varphi(E)$ in unit $\mathrm{cm}^{-2} . \mathrm{s}^{-1}$ ) for the first of January of the year 2016, 17, 18 and 19 are presented in Fig. 4. The relative proportions of the each energy domain (thermal, epithermal, evaporation and cascade) are relatively invariant (Table I). This remark concerns four days for which solar conditions are quiet.

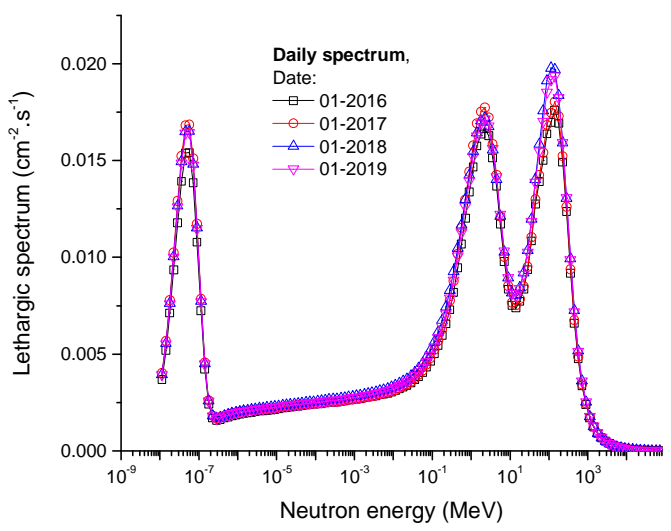

Table I: Total neutron flux and contributions in \% of thermal (Th.), epithermal (Epi.), evaporation (Eva.) and cascade (Cas.) neutron, for the OI January of 2016, 17, 18 and 19.

\begin{tabular}{l|l|lllll}
\hline & \multicolumn{4}{|l|}{ Total flux } & \multicolumn{4}{l}{ Contribution (in \%) } \\
& \multicolumn{1}{|c|}{ cm $^{2} / \mathbf{s}$} & Th. & Epi. & Eva. & Cas. \\
\hline $\mathbf{0 1 / 0 1 / 2 0 1 6}$ & 0.165 & 17.6 & 19.5 & 34.3 & 28.6 \\
$\mathbf{0 1 / 0 1 / 2 0 1 7}$ & 0.175 & 18.3 & 19.1 & 34.7 & 28.0 \\
$\mathbf{0 1 / 0 1 / 2 0 1 8}$ & 0.172 & 17.4 & 19.4 & 33.9 & 29.3 \\
$\mathbf{0 1 / 0 1 / 2 0 1 9}$ & 0.178 & 17.6 & 19.2 & 33.9 & 29.4 \\
\hline
\end{tabular}

Fig. 4. Total corrected (by water vapor and atmospheric pressure) neutron flux from January 2016 to January 2019, issued from measurements and obtained by calculations using ATMORAD calculations and considering solar potential extract from Oulu data.

\subsection{Short-term analysis: example of September 2017 solar event}

During the first days of September 2017, the Sun was very active inducing solar flares. After a series of major flares including $27 \mathrm{M}$-class and $4 \mathrm{X}$-class flares, the solar active region had produced an X9.3 flare on the 6th September. The solar activity decreased on the 9th and 10th September. Bright gamma-ray emission from the X8.2 class solar flare was recorded on the 10th September 2017 by the Large Area Telescope and Fermi Gamma-ray Space Telescope [11]. This flare was characterized by a SEP event with high-energy particles, producing nuclear 
cascades in the atmosphere and detected on the ground. This flare was the second GLE (\#72) of the current solar cycle. Fig. 5 presents the temporal series relative to (a) the water vapor absolute humidity (ground, $+100,+250$ and $+500 \mathrm{~m}$ ), (b) the IWV parameter, (c) the uncorrected and corrected (pressure and pressure + water vapor) hourly count rate obtained for the 7'Tungsten detector and (d) the total corrected neutron flux during the observed solar event (6 - 13 September 2017).

As stated above, the data show that the Concordia environment can be therefore considered extremely dry during the solar event. Two FDs can be identified in Fig. 2(d). The second FD is composed by diurnal variations due to the anisotropy and superposed on the recovery step. It is a typical multi-step FD [12], the amplitudes of steps are in the order of $3 \%$ and $11.3 \%$, respectively.

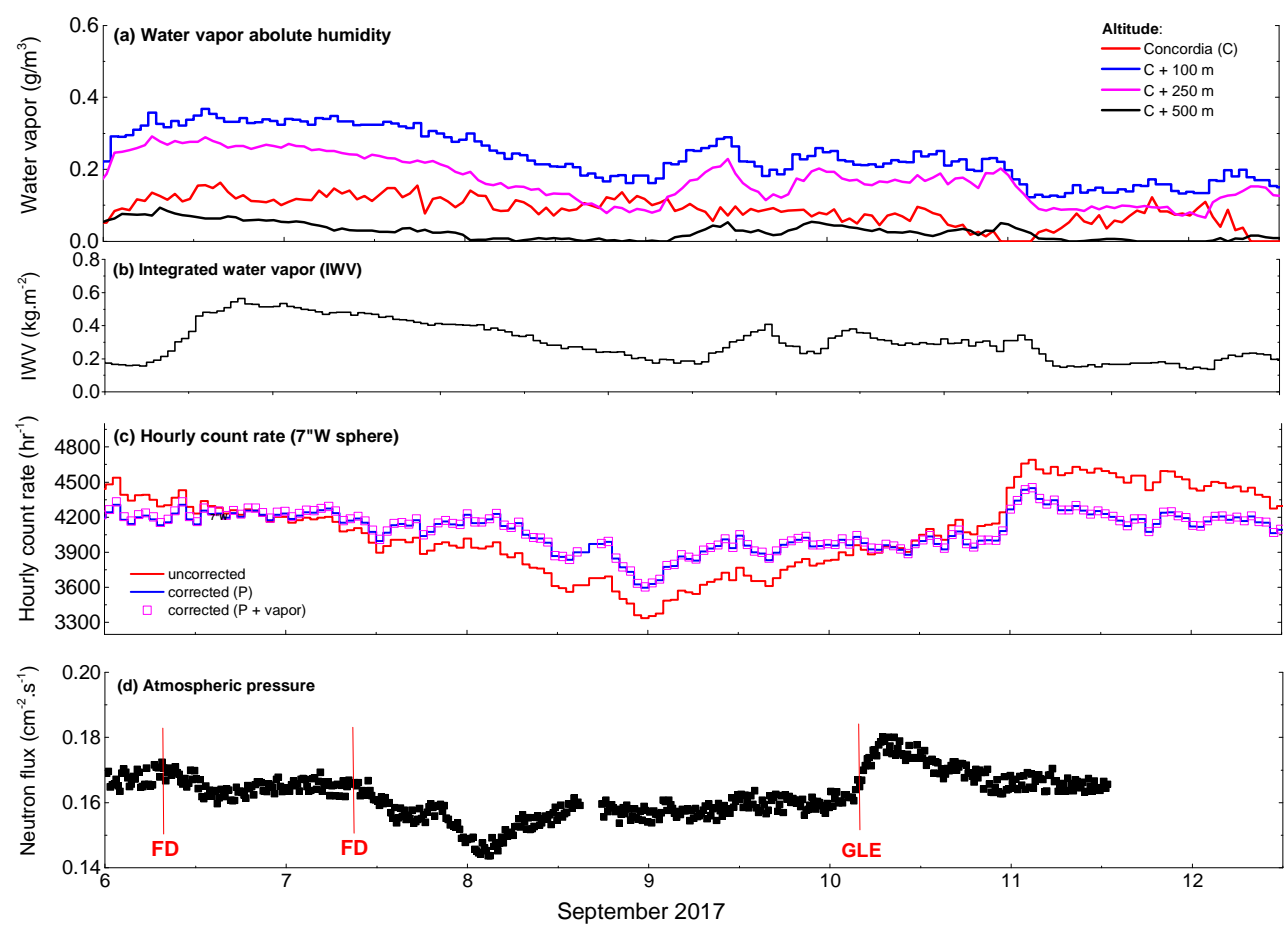

Fig. 5. (a) water vapor absolute humidity (ground, $+100,+250$ and $+500 \mathrm{~m}$ ), (b) the IWV parameter (water vapor integrated along the vertical from the surface to the tropopause), (c) the uncorrected/corrected hourly count rate (7'Tungsten sphere) recorded in Concordia during solar events and (d) the total neutron flux.

Complementary analyses [13] investigate the flux variations for each neutron energy domain and during the solar event including FD and GLE. During FD, fluxes relative to the four energy domains decrease according to a similar factor. However, during GLE, the thermal, epithermal and evaporation neutron populations increase more than cascade neutrons. The thermal peak presents a change in amplitude resulting from a change in the evaporation neutron population. Evaporation neutrons are moderated in the environment producing thermal neutrons and the increasing thermal neutrons are directly impacted by the increasing of evaporation neutrons component. 


\section{Conclusion}

First four year of measurements of the energy spectrum of CR on the Concordia Station in Antarctica are presented. The particularity of these analyses is that the data from the HAMSTRAD project were used to take into account the environmental and systematic effects. Thus, temporal series of integrated water vapor, liquid water path, atmospheric pressure, neutron fluxes and spectra were presented. Long- and short-term analyses were presented, in particular during the solar event of September 2017. Simulations allow to show that variations observed in the long-term are mainly attributable to the solar activity. The relative proportions of each energy domain (thermal, epithermal, evaporation and cascade) are relatively invariant except during GLE event for which thermal, epithermal and evaporation domains are the most impacted.

\section{References}

[1] P. Ricaud, et al., HAMSTRAD-Tropo, A 183-GHz Radiometer Dedicated to Sound Tropospheric Water Vapor Over Concordia station, Antarctica, IEEE Transaction On Geoscience and Remote Sensing, 48(3), 1365-1380, 2010

[2] P. Ricaud, et al. Review of tropospheric temperature, absolute humidity and integrated water vapour from the HAMSTRAD radiometer installed at Dome C, Antarctica, 2009-14. Antarctic Science, 27, 598-616, 2015

[3] G. Hubert, Analyses of cosmic ray induced-neutron based on spectrometers operated simultaneously at mid-latitude and Antarctica high-altitude stations during quiet solar activity. Astroparticle Physics Journal, 83, 30-39, 2016.

[4] H. Cane, Coronal Mass Ejections and Forbush decreases. Space Science Reviews, 93, 55-77, 2003.

[5] G. Hubert et al., Modelling of ground albedo neutrons to investigate seasonal cosmic-ray-induced neutron variations measured at high-altitude stations. J. Geophys. Res. Space Physics, 121, 186-201, 2016.

[6] R. Rosolem, et al., The effect of atmospheric water vapor on neutron count in the cosmic-ray soil moisture observing system, Journal of Hydrometeorology, 14, 1659-1671, 2013.

[7] G. Leuthold, et al., Long-term measurements of cosmic ray neutrons by means of a Bonner Spectrometer at Mountain altitudes - First results. Radiation Protection Dosimetry, 126(1-4), 206511,2007

[8] G. Hubert, et al., Radiation effects investigations based on atmospheric radiation model (ATMORAD) considering GEANT4 simulations of extensive air showers and solar modulation potential. Radiation Research, 184(1), 83-94, 2015.

[9] L. Gleeson, W. Axford, Solar Modulation of Galactic Cosmic Rays. the Astrophysical Journal, 154, 1011-1027. 1968.

[10] Oulu, Cosmic Ray Station, https://cosmicrays.oulu.fi/

[11] W. Atwood, et al., The Large Area Telescope on the Fermi Gamma-Ray Space Telescope Mission. The Astrophysical Journal, 697, 1071-1102, 2009.

[12] A. Bhaskar, et al., Role of solar wind speed and interplanetary magnetic field during two-step Forbush decreases caused by Interplanetary Coronal Mass Ejections. Astrophys Space Sci, 361, 242-255, 2016

[13] G. Hubert et al., Analysis of the Forbush Decreases and Ground-Level Enhancement on September 2017 using Neutron spectrometers operated in Antarctic and mid-latitude stations, Journal of Geophysical Research: Space Physics, 124(2), 2019. 